\title{
问

10-17-2014

\section{Using Design Hierarchy in a Linear Circuits Class to Illustrate the Scientific Method as a Human Invention}

Douglas DeBoer

Dordt College, douglas.deboer@dordt.edu

Follow this and additional works at: https://digitalcollections.dordt.edu/faculty_work

Part of the Educational Methods Commons, Electrical and Computer Engineering Commons, and the Engineering Education Commons

\section{Recommended Citation}

DeBoer, D. (2014). Using Design Hierarchy in a Linear Circuits Class to Illustrate the Scientific Method as a Human Invention. Retrieved from https://digitalcollections.dordt.edu/faculty_work/59

This Conference Proceeding is brought to you for free and open access by Dordt Digital Collections. It has been accepted for inclusion in Faculty Work Comprehensive List by an authorized administrator of Dordt Digital Collections. For more information, please contact ingrid.mulder@dordt.edu. 


\title{
Using Design Hierarchy in a Linear Circuits Class to Illustrate the Scientific Method as a Human Invention
}

\begin{abstract}
There are various ways to classify academic studies. One might make a "two cultures" division, separating academic studies into the humanities and sciences. Or one might have three divisions: humanities, social sciences, and natural sciences. Or one might choose to classify academic studies along the lines of traditional academic disciplines such as theology, law, art, music, economics, social studies, languages, political studies, history, psychology, biology, physics, chemistry, math, etc. In our era of global commerce, where does engineering fit in these types of classification? What should engineers study? In a 2008 presidential debate, candidate Barack Obama said that, "Ensuring that the U.S. continues to lead the world in science and technology will be a central priority for my administration." Candidate John McCain said that public policy ought to be based upon sound science.

(http://www.sciencedebate.org/debate08.html) Others might say they "believe in global warming" or "evolution," or some other topic of current interest having a rather obvious basis in science. Why is there such agreement that science should be foundational in public policy? Specifically, how does science fit into an engineering education? Scientific theories are human formulations intended to describe and predict the behavior of the natural world around us. Engineering work relies on scientific theories to be sure, but also on many other academic disciplines. To train engineering students as "renaissance people" requires that our students understand engineering as a multidisciplinary subject and a human endeavor. Any engineering course ought to help students understand the breadth and depth of engineering. This paper illustrates some examples of how this can be done for a linear circuits class. Hopefully by seeing an example, the reader can generalize the ideas to other engineering courses. The hierarchical structure of engineering subjects, and specifically, linear circuits, naturally lends itself to an illustration of how the science of linear circuits is humanly constructed. The foundation of this science can be traced back to axioms. Some specific aspects of the scientific method that can be elaborated on in the context of linear circuits are the hierarchical nature of the scientific method, the axiomatic foundation of these hierarchies, the limited scope of scientific theories and its utilitarian goals, essentially to predict the future if given enough of the right information about the past and present. In contrast, the universe does not exist in hierarchies, it is wholistic, and always has unpredictable aspects. Linear circuits are also wholistic. When constructed and actually used they have limitations and even in some cases unpredicted behaviors which the scientific theories of linear circuits do not fully explain. Such understanding of the scientific method as a human invention is essential to relating ones engineering (or science) to the culture around us, so that our work is responsive to real needs and is recognizably beneficial.
\end{abstract}

\section{Keywords}

electrical engineering, linear circuits, educational methods, engineering students, Dordt College, ASEE

Disciplines

Educational Methods | Electrical and Computer Engineering | Engineering Education

\section{Comments}

Paper presented at the 2014 American Society for Engineering Education (ASEE) North Midwest Section Conference held on the campus of the University of lowa in lowa City, lowa, October 16-17, 2014.

Copyright $@ 2014$, Douglas De Boer.

This conference proceeding is available at Dordt Digital Collections: https://digitalcollections.dordt.edu/faculty_work/ 


\title{
Using design hierarchy in a linear circuits class to illustrate the scientific method as a human invention.
}

Dr. Douglas De Boer, PE

Dordt College, Sioux Center, Iowa

\begin{abstract}
There are various ways to classify academic studies. One might make a "two cultures" division, separating academic studies into the humanities and sciences. Or one might have three divisions: humanities, social sciences, and natural sciences. Or one might choose to classify academic studies along the lines of traditional academic disciplines such as theology, law, art, music, economics, social studies, languages, political studies, history, psychology, biology, physics, chemistry, math, etc. In our era of global commerce, where does engineering fit in these types of classification? What should engineers study? More fundamentally, why should engineers study science?
\end{abstract}

In a 2008 presidential debate, candidate Barack Obama said that, "Ensuring that the U.S. continues to lead the world in science and technology will be a central priority for my administration." At the same debate, Candidate John McCain said that public policy, "should be based upon sound science." 1 Within the field of engineering education, there are those who look to scientific research on the topic of engineering education as key to achieving meaningful improvements to engineering education. ${ }^{2}$ Within the general public there are those who might say they "believe in global warming” or "evolution," or some other topic of current interest having a rather obvious basis in science. Why is there such general agreement that science should be foundational in so many aspects of life, including engineering education?

Scientific theories are human formulations intended to describe and predict the behavior of the natural world around us. Engineering work relies on scientific theories to be sure, but also on many other academic disciplines. To train engineering students as "renaissance people" requires that our students understand engineering as a multidisciplinary subject and a human endeavor. Any engineering course ought to help students understand the breadth and depth of engineering as well as the foundational role of science. This paper illustrates some examples of how this can be done within a linear circuits class. Hopefully by seeing an example, the reader can generalize the ideas to other engineering courses.

The hierarchical structure of engineering subjects, and specifically, linear circuits, naturally lends itself to an illustration of how the science of linear circuits is humanly constructed. Some specific aspects of the scientific method that can be elaborated on in the context of linear circuits are the hierarchical nature of the scientific method, the axiomatic foundation of these hierarchies, the limited scope of scientific theories and its utilitarian goals, essentially to predict the future if given enough of the right information about the past and present. In contrast, the universe does not exist in hierarchies, it is wholistic, and always has unpredictable aspects. Linear circuits are also wholistic. When constructed and actually used they have limitations and even in some cases unpredicted behaviors which the scientific theories of linear circuits do not fully explain. Such understanding of the scientific method as a human invention is essential to relating ones 
engineering (or science) to the culture around us, so that our work is responsive to real needs and is recognizably beneficial.

\section{Science and Engineering Defined}

A dictionary definition of science is, "The intellectual and practical activity encompassing the systematic study of the structure and behavior of the physical and natural world through observation and experiment."3. The same dictionary defines the scientific method as, "consisting in systematic observation, measurement, and experiment, and the formulation, testing, and modification of hypotheses." 4 These definitions of science and the scientific method do indeed make reasonable sense of the statements of President Obama, John McCain, and others. Obviously, these people considered science and the scientific method as knowledge and principles used by people to bring about a desirable future. The importance of applying science in these contexts rests in its accepted utilitarian value. People could make other choices. For example, someone might advocate that public policy, or some other matter, should be based upon religion instead of, "sound science.” Or one might choose to base some matter upon economics or aesthetics instead of, "sound science.” There are, of course, many possible fundamental bases upon which to rest a decision. The choice of a basis, or some weighting of several bases, is a humanly made choice.

Consider now how these definitions and applications of science relate to engineering. A dictionary definition of engineering is, "The branch of science and technology concerned with the design, building, and use of engines. .." (The word "engineering” might also refer to certain works or an occupation or an artful design to bring something to fruition. $)^{5}$ The concept of an "engine" in this definition is intended to be taken broadly, meaning to include machines, structures, and various technically complicated physical systems, etc. Although the concept of engineering as having to do with the design of "engines" is rather memorable, the true core of this definition of engineering is that it is a branch of science and technology, or in other words, it involves the application of the scientific method, toward the design, building and use of "engines." This definition highlights science to such a degree as to imply that the application of the scientific method is practically the only activity involved in doing engineering! It is no wonder that in light of this overly-narrow definition of engineering the public has difficulty understanding what engineers do. Indeed, engineers get involved in government and politics in order to create regulations and standards, create building codes and other quisi-legal entities. They are also employed by government to standardize the weights and measures that are the basis of all commerce (e.g. Bureau International des Poeds et Mesures in France, and The National Institute of Standards and Technology in the U. S.). They write and provide expertise on patents, and in sort, they do quite a lot of work that goes beyond simply making hypotheses and testing them (the scientific method) and also goes beyond even a generously broad interpretation of, “designing, building, and using engines.” The full breadth of engineering activities is rooted in a response to the technological desires of a culture. In order to respond appropriately within a cultural context, engineers need a reasonable breadth of studies in all academic disciplines, with a recognition that the natural sciences have a foundational role to the work of engineers. 
In their university courses, engineering students should become aware (if they are not already aware) of the cultural motivation behind doing engineering work. It is in the context of a relationship with the culture they are working in that engineering students will find a sense of joy and fulfillment in their studies. Merely “designing, building, and using engines” for no particular purpose offers little spiritual enrichment beyond the aesthetic satisfactions of the sounds, textures, and other characteristics of “engines" themselves. While that narrow aesthetic satisfaction may be adequate for some students, a more full-orbed concept of engineering work will bring significantly more satisfaction for most students.

\section{Teaching Linear Circuits}

Most engineering courses rely on hierarchies of knowledge, and a linear circuits course is no exception. By discussing hierarchies with the students one can draw attention to the human activity that organized the body of knowledge (science) used in the course. A key concept that needs to be communicated to students is that science, as a body of knowledge, is a result of human activity. Humans, being what we are, do not come up with a perfect body of knowledge on a first attempt. Human activity itself is also not exclusively scientific, perhaps even if we are tying to produce a scientific experiment. In our choices of what to study and what not to study, and in what hypotheses we choose to consider, and even in our choices of what evidence is required to prove a hypothesis, humans make incremental progress as time passes, with occasional mistakes included. These choices and decisions are themselves not scientific. They are influenced by economic conditions and social opportunities, and also fundamental belief structures, including conceptions of reality. In some cases the choice of what to study can possibly even be influenced by fads and traditions. Helping students understand this helps students distinguish between various conceptions of truth and cultural biases. This point can be illustrated by an example showing how we abstract reality in order to create a hypotheses for scientific investigation.

\section{Scientific Abstraction Exemplified.}

Imagine that the designer of an automobile says, "The electrical consumption of this car needs to be reduced." (Perhaps the alternator is not large enough to supply the present load and prior decision-making has eliminated the possibility of upgrading to a larger alternator.) At this point, in order to gain an understanding of where electrical consumption might be reduced, the engineer might make in inventory of the current consumption of the various parts of the automobile. As simple as this process might be, the values of the engineer will come into play. For example, the car's electrical loads might be divided into categories such as, lighting system, ignition system, and everything else. But why not instead divide the categories into entertainment systems, safety systems, and motive systems? The choice of categories is inherently non-scientific and expressive of what the engineer values. An engineer who values economy of operation would likely make a different choice of hierarchies and categories than an engineer who values the aesthetic aspects of the car. An engineer concerned with the ultimate environmental impact of the car might make still a different choice of hierarchy and categories. 
One might argue that none of the categorizations in the above example are detailed enough. Indeed, one can make more and different categories to get a more detailed perspective of the situation. For example, within the "lighting" category, one could itemize "exterior," "cabin," and "other lights." Then within the "exterior" category one could itemize, "headlights," "tail lights," "turn signals," etc. There is practically no limit to the amount of detail one could imagine itemizing in a hierarchical fashion. (The lights could be further classified by high and low beam, color, incandescent vs. LED, etc. Incandescent lamps can be categorized by base styles, internal gases, filament metallurgy, etc. and etc.) As more and more levels are added to the hierarchy and as more categories are introduced, the hierarchy more completely characterizes the electrical consumption of the car. The picture revealed by the hierarchy becomes a more wholistic representation of the real situation. However, as a matter of utility and economy, the engineer will use as few hierarchical levels and categories as are practical. Engineers must deliberately omit some, or even a lot of detail from consideration. This is necessary in order to focus attention on the need at hand. The choices of what to include and what to omit from our attention are not scientifically made choices. Science comes after these prior choices of hierarchies and categories are made.

When the final decisions are made and the product (the automobile in this example) is built, the actual behavior of the product is wholistic. That is, the product acts as nature would have it act, not necessarily as the engineering predicted. It is possible that the abstractions (what to consider and what to omit) and the hierarchies used in the engineering were not adequately descriptive to achieve the desired final behavior.

Abstraction, then, is the human process of identifying patterns in real situations by paying attention to only a small but relevant portion of the available detail. The scientific method is a process based upon a prior abstraction which is humanly invented. In this sense, science, the scientific method are just some of the types of activities engineers engage in. An important human activity that comes prior to doing science is the process of abstraction. In any process of abstraction important expressions of value come into play.

\section{Some Hierarchies in Linear Circuits}

Most linear circuits courses start with a chapter or two of basic definitions of electrical quantities such as current, voltage, electrical power, resistance, nodes, branches, etc. Students need to realize that all of these definitions are culturally constructed and not simply handed down to us, as if nature could talk.

Consider the definition of current, as might typically be encountered in a linear circuits course. Any linear circuits textbook definition will suffice for our consideration since they are all similar. For example, Hayt, Kermmerly and Durbin's textbook states, "We define the current at a specific point and flowing in a specified direction as the instantaneous rate at with net positive charge is moving past that point in the specified direction." ${ }^{6}$ One could introduce this definition by asking students to define the current flow in a river. Students will have various conceptions 
of this concept. For example, a student familiar with river navigation will be likely to discuss the speed at which the surface of the water moves relative to the surrounding landscape. Indeed, knowing the "current" in the river is, say, 3 miles-per-hour, is very useful knowledge for navigating the river. However a student concerned with water resources management and conservation might be more interested in the volume-rate of the flow of the river, say in gallonsper-hour. This conception of the "current" in the river makes it easier to understand the impact of tributaries on the river, and the impact on the river of pollution from the tributaries. The two different conceptual definitions of the current flow in a river are not reducible to one definition. Factors such as the width and depth and the velocity profile within the river need to be known to convert from one to the other, and these factors vary from one situation to another.

By discussing these basic definitions one can show how the definition of electrical current, as presented in the textbook, is rooted in a cultural desire to apply electrical circuits to achieve certain technologically-rooted desires (e.g. lighting, communication, transportation, etc.) Given different goals, a different definition of electrical current could conceivably be more appropriate, even if a different definition would be highly inappropriate for the linear circuits course! However, for clarity we typically give different names to different definitions or conceptions of a quantity. For example, in a solid-state devices course current density may be much more useful than current flowing "past a point." The definitions themselves are not scientifically proven hypotheses. Rather, they are axiomatic human inventions with considerable utility. They arise out of a process of abstraction.

After basic definitions are presented in a linear circuits course, some theorems or laws, such as Kirchhoff's Current and Voltage Laws, are presented. This is another humanly invented layer of abstraction. These theorems can be proven via the scientific method, but the formulation of the original hypotheses are humanly devised and rooted in utilitarian goals.

The linear circuits course proceeds through a list of increasingly abstract hierarchically structured theorems and laws. Typically these would include superposition, Thevenin's and Norton's theorems, nodal and mesh analysis, inductors and capacitors, phasors, and Fourier Series. Supplementing the textbook with the cultural-historical concepts behind these humanly invented theories and methods makes the course more interesting and fulfilling, giving students motivation for further study.

Note that in electrical technology courses (in contrast to engineering courses) a different hierarchy of knowledge is usually applied to this subject. It is usually organized around the type of circuit encountered, such as DC or resistive circuits, electromagnetism, and AC circuits (meaning sinusoidal circuits). An expression of this hierarchy might be found in the table of contents of a typical textbook. A different ultimate goal for the utility of the course has resulted in a different hierarchy of knowledge. (One evidence of this could be the table of contents of an engineering technology textbook on circuits, for a specific example, the textbook by Robins and Miller could be taken as typical of the genre. ${ }^{7}$ ) 


\section{The foundational role of science in engineering}

Recall the earlier example of an engineer desiring to reduce the electrical consumption of an automobile. Table 1 below expresses a possible example hierarchy that might relate to the situation.

Table 1. Example of Hierarchy

\begin{tabular}{|l|l|}
\hline A Hierarchy & Other items “at the same level” \\
\hline $\begin{array}{l}\uparrow \text { (One could imagine more over-arching } \\
\text { categories in higher levels of hierarchy.) }\end{array}$ & Utility distribution systems (water, electricity) \\
\hline $\begin{array}{l}\text { Transportation system } \\
\text { (including air, rail, truck, auto, ship. . . ) }\end{array}$ & shipping lanes, air-traffic control \\
\hline Road and highway system & truck, ship, airplane \\
\hline Automobile & fuel system, suspension, exhaust, engine \\
\hline Electrical system in an automobile & ignition system, entertainment system \\
\hline Lighting system in an automobile & headlights, courtesy lights \\
\hline Tail light system & parking lights, license plate light, \\
\hline Right-turn indicator & turn signal switch, turn signal wiring harness \\
\hline flasher for turn indicator & $\begin{array}{l}\text { bi-metallic heater in electro-mechanical turn } \\
\text { signal flasher }\end{array}$ \\
\hline $\begin{array}{l}\text { Bi-metallic element in electro-mechanical turn } \\
\text { signal flasher }\end{array}$ & $\begin{array}{l}\text { anchor for bi-metallic element, sound resonator } \\
\text { attached to bi-metallic element }\end{array}$ \\
\hline $\begin{array}{l}\text { Contact point on bi-metalic element in turn } \\
\text { signal flasher }\end{array}$ & \\
\hline$\downarrow$ (One could imagine even more detail by \\
adding lower levels of hierarchy.)
\end{tabular}

Observe that as one moves up in the hierarchy the straight-forward application of the scientific method to answer questions becomes increasingly difficult. What makes a transportation system good? For that matter, what makes an automobile good? Different people will value different aspects.

Conversely, as one moves down the levels of the hierarchy the design issues become further abstracted from the ultimate cultural utility of the engineering work done. Designing a highly efficient contact point for a bi-metallic-type turn signal flasher hardly seems culturally important compared to designing a transportation system. Yet the design of internal parts in the turn signal flasher is an enabler of the ultimate cultural objective.

At the lower levels of the hierarchy the application of the scientific method becomes more obvious. What makes a contact point good? Without a whole lot of debate, one person or a small team of people can propose a few hypotheses and extensively test them. This is the sense in which science plays a foundational role in engineering design. 


\section{Conclusion}

In engineering work, science and the scientific method have their most significant roles at lower levels in the hierarchy of the design. In that sense, science is foundational to engineering design. On the other hand, science is not the final arbiter at any level of hierarchy. The application of the scientific method is a human activity depending on abstraction, and thus differently motivated people may come up with different solutions or analyses. The various types of knowledge employed in an engineering project, including the scientific method, but also including political, mathematical, aesthetic, legal, and many other forms of knowledge, are human responses to a cultural situation. Joy and fulfillment are enhanced in engineering work when these cultural connections are understood. These concepts can be incorporated into engineering courses, in particular, into a linear circuits course.

\section{Bibliography}

1. Science Debate 2008, http://www.sciencedebate.org/debate08.htm, accessed 7/31/2014.

2. Felder, R. M., Hadgraft, R. G. (2013). Educational Practice and Educational Research in Engineering: Partners, Antagonists, or Ships Passing in the Night? Journal of Engineering Education, 102(3), 339-345.

3. Oxford US English Dictionary, accessed online July 28, 2014, http://www.oxforddictionaries.com/us/definition/american_english/science

4. Oxford US English Dictionary, accessed online July 28, 2014, http://www.oxforddictionaries.com/us/definition/american_english/scientific-method

5. Oxford US English Dictionary, accessed online July 28, 2014, http://www.oxforddictionaries.com/us/definition/american_english/engineering

6. Hayt, W. H., Kemmerly, J. E, Durbin, S. (2012) Engineering Circuit Analysis, McGraw-Hill, p12.

7. Robins, A. H., Miller, W. C., (2000) Circuit Analysis Theory and Practice, Delmar. 